3

\title{
Representing Uncertainty in Natural Hazard Risk Assessment with Dempster Shafer
}

\section{(Evidence) Theory}

Wendy Ballent, Graduate Student Researcher, Dept. of Civil, Environmental, and Architectural Engineering, University of Colorado Boulder, Boulder, CO 80309 (email: wendy.ballent@,colorado.edu )

Ross B Corotis, Professor, Dept. of Civil, Environmental, and Architectural Engineering, University of Colorado Boulder, Boulder, CO 80309 (email: ross.corotis@colorado.edu )

Cristina Torres-Machi, Assistant Professor, Dept. of Civil, Environmental, and Architectural Engineering, University of Colorado Boulder, Boulder, CO 80309 (email: cristina.torresmachi@colorado.edu )

\section{ABSTRACT}

This paper explores and develops different mathematical frameworks to address the representation of inherent uncertainties such as those often involved in the assessment of natural hazard risk for the built environment. To date, little exploration has been performed of such theories, inhibiting the progress and use of these potentially well-suited frameworks, especially to applications for expert evidence in the field of sustainable and resilient infrastructure. One such framework, Dempster-Shafer Theory, allows the combination of multiple expert beliefs while considering uncertainties that are often ingrained in this field. In cases such as seismic hazards, for which structural vulnerability and structural damage are evaluated in a case-by-case scenario, subjective assessments are not only useful but necessary. This research performs a rigorous exploration to determine the behavior and trends of Dempster-Shafer Theory, including a mathematical proof of asymptotic behavior, in an attempt to both (a) understand how this framework handles confidence, ignorance, and combined beliefs, and (b) encourage the use of more natural frameworks in cases that involve uncertainty. The results of this exploration suggest that probability may not be the most natural framework in which to quantitatively incorporate the involved uncertainty. Ignorance and evidence-based assessments may be better represented using Dempster Shafer Theory. 
Risk assessment is an integral component of modern engineering and hazard mitigation, but presents a mathematical obstacle due to the inherent uncertainties involved in such evaluations. The field of natural hazard assessment for infrastructure often requires assessments that are inherently subjective in nature, as no structure or location is exactly the same. At any given project, a limited number of experts may be available to provide their risk evaluations using varying amounts of evidence and information. A variety of frameworks could be considered when handling such uncertainties.

Probability often provides a reliable structure in such situations, where an educated guess can be made based on the outcome of previous similar occurrences and professional judgment. There are many circumstances, however, in which the sources of uncertainty and knowledge of the modeling are not well represented within the constraints of probability theory, as will be discussed below. Such circumstances include ignorance (when there is limited amount of data), varying degrees and sources of confidence, and situations that are not repetitive enough to use previous data to create robust frequency-based probability, either directly or through subjective assessment.

The "subjectivists" offered an interpretation of probabilities as a "degree of belief"; the probability of an uncertain event as a measure of one's belief about its occurrence (Vick 2002 p. 20). As stated by Melchers (1999), "a subjective probability estimate reflects the degree of ignorance about the phenomenon under consideration". The class of subjective probabilities, or Bayesian degrees of belief, allows for a broad context of Probability Theory, justified not necessarily by the objective or frequentist basis, but to single occurrence events in the form of a measure of one's uncertainty about a particular event. As such, judgments manifested in the form of subjective probabilities can be manipulated with the axioms of Probability Theory. Although this offers a powerful framework for systematically incorporating uncertainty into almost any problem, subjective probabilities cannot distinguish between situations such as known equal outcomes and complete ignorance. In addition, whether probability 
51 estimates are based on objective (frequentist) models or subjective models, they must by definition obey

52 the axioms of probability theory. Further, judgments treated with a probabilistic model suggest there is

53 precise information not only about the event itself, but also its contrary, which may not be appropriate in

54 cases with little or partial knowledge. For instance, suppose a piece of equipment could have been

55 manufactured in country A or country B, but no other. Limited evidence leads to assigning a belief or

56 confidence of $20 \%$ that its origin is country A, and a belief or confidence of $30 \%$ that it came from

57 country B. That leaves a 50\% uncertainty or ignorance of belief assignment regarding the country of

58 origin. These realizations inspired research into a broader conception of uncertainty, exploring important

59 facets of uncertainty that are not probabilistic in nature. These other forms of characterizing uncertainty

60 have received very limited attention in the area of structural risk and vulnerability, and it is now apparent

61 that a complete paradigm shift in embodying uncertainty is needed for more robust and resilient theories

62 of structural and community vulnerability (Corotis 2015).

63 There has been a significant amount of research that explores the relevance and applicability of other

64 mathematical theories dedicated to the treatment of uncertainty, but many of these methods remain only

65 partially developed and not investigated in terms of their applicability to the field of civil engineering

66 (see, for example, Adeli 1988, Ayyub 1998 and 2001, Ayyub and Klir 2006, Booker and Ross 2011, Klir

67 2006, Ross 2010 and Shafer 1976 and 1987). The research presented in this paper examines the

68 characteristics of uncertainty beyond traditional probabilistic modeling, and is motivated by these primary

69 objectives: (i) introducing appropriate roles for uncertainty theories beyond Probability Theory and their

70 associated relevance, (ii) developing a deeper awareness and understanding of uncertainty's potential role

71 within civil engineering as applied to risk assessment of infrastructure, and (iii) creating a more

72 comprehensive uncertainty model for future research in this field. The motivation for new approaches is

73 not intended to challenge the fundamentals of Probability Theory, but to present different mathematical

74 models, which may be relevant in a variety of contexts. The first objective is very important because the

75 confluence of geophysics and structural engineering with aspects of environment, economics, and social 
and political capital means that such disparate facets are likely to require increased usage of expert opinions and beliefs. These issues reflect very different natures and sources of uncertainty, and in this paper we will show how generalized uncertainty provides some powerful approaches, but also some unexpected results that as far as the authors know have never been explored. Regarding the second objective, the first example in this paper will show the use of belief theory in a seismic damage assessment, demonstrating its use in a practical situation involving risk analysis to the built environment. Finally, previously unexplored trends, sensitivities and a mathematical derivation serve to alert potential users of some of the subtleties of generalized uncertainty.

\section{Literature Review}

\section{Uncertainties in Risk Assessment}

In 1976, Glenn Shafer presented his work and the work of his mentor, Arthur Dempster, in “A Mathematical Theory of Evidence" (Beynon et al. 2000; Shafer 1976). This work features a Theory of Evidence in which belieffunctions can be formalized from a degree of belief based on available information and knowledge, termed beliefs and plausibilities (Yager and Liu 2008). As the works became known to the artificial intelligence community, the theory fell under the name of the Dempster-Shafer Theory of evidence, or commonly, Evidence Theory (Shafer 1976). Since Dempster-Shafer Theory's origination, it has been evaluated as a potential alternative to classical, frequentist, and subjective probability. Classical, frequentist probabilities are conceptualized as the number of outcomes resulting in the specified event divided by the total number of outcomes if the situation were repeated (technically as that number approaches infinity), while subjective is entirely on the assigner's degree of belief. There is a clear demand in the world of science and engineering for a method of risk assessment that addresses the inevitable uncertainties of the field (Cooke 1991).

\section{Generalized Information Theory}


Recent theories that extend beyond probability include imprecise probabilities, probability-bound

analysis, Possibility Theory, and Dempster-Shafer Theory. Motivated by the emergence of various mathematical models for handling uncertainty and partial information of different types, a new area of study termed Generalized Information Theory (GIT) was formally introduced in the early 1990s (Ayyub 1998; Klir 2006; Ross 2010). This area of study is aimed at formally recognizing and systematically dealing with the nature and scope of uncertainty and its association with partial knowledge. In other words, GIT is concerned with the development of uncertainty theories.

GIT expands Probability Theory in two dimensions by including non-additive probability measures and fuzzy sets (rather than Classical Set Theory) (Klir 2006). This paper focuses on the former, specifically the generalization of the uncertainty associated with the assignment of an element. This area of study falls under the Theory of Monotone Measures (Klir and Smith 2001; Wang and Klir 2009).

\section{Monotone Measures}

Monotone measures broaden the mathematical framework of Probability Theory. There are several classes of monotone measures that generalize the notion of uncertainty in the assignment of an element $(x)$, out of a universe $X$, to a particular set (․ㅡ). Measures include possibility/necessity measures, Sugeno $\lambda$-measures, belief/plausibility measures, interval-valued probability distributions, and imprecise probabilities (general lower and upper probabilities). Of these, possibility/necessity measures, belief/plausibility measures, and imprecise probabilities are among the most promising for the evaluation of uncertainty in a structural or community risk, reliability, vulnerability, and resilience context. In the context of classical probability, the assignment of an element $x$ to the set $\underline{\mathrm{A}}$ is typically interpreted as a matter of likelihood or chance, or in the context of subjective probabilities, as a degree of certainty.

Monotone measures generalize this interpretation, typically associating notions of incomplete information with 'evidence' pertaining to $x$.

Mathematically, a monotone measure---denoted $g(\underline{\mathrm{A}})$---is a mapping to the power set (a set of beliefs on any available event or event combination) on the unit interval. The value assigned to $g(\underline{A})$ is an 
expression of the degree of support for the belief that an element $x$ belongs to a given crisp subset $\underline{A}$ (Ross

127 2010). Two axioms for monotone measures establish the boundary conditions for any monotone measure:

$128 g(\varnothing)=0$ signifies no degree of support in the null set and $g(X)=1$ indicates complete belief for the entire

129 universe. Another requirement states that the evidence supporting $\underline{B}$ must be at least as great as the

130 evidence assigned to $\underline{\mathrm{A}}$, when $\underline{\mathrm{A}}$ is completely contained in $\underline{\mathrm{B}}$, the statement of monotonicity.

Probability Theory satisfies the axioms of monotone measures, but in addition must satisfy the

additivity requirement, which is a critical restriction for the use of expert opinions. As demonstrated by subsets of $\mathrm{X}$ if bound within the disjoint set as shown below:

$$
P(\underline{A} \cup \underline{B})=P(\underline{A})+P(\underline{B})
$$

Equation (1) requires that any information provided about element or set ' $\underline{A}$ ' also provides contrary

137 evidence about the complementary event or set ' $\overline{\mathrm{A}}$ '. In Probability Theory, uncertainty is represented by

this single probability measure. If either the probability of an event or set or the probability of its negation

139 (or complement) is known, the additivity requirement guarantees that the probabilities of both are known.

140 In the case of evidence theory, one expert might be prepared to assign a certain degree of evidence

141 (sometimes conveniently viewed as confidence or belief), for instance, that a particular structure has been 142 rendered uninhabitable following an earthquake. As an example, let this event be designated A, and let 143 this belief be $30 \%$. Because there is a lot of uncertainty and unknown about the details of the building's 144 condition, this same expert might be prepared to assign a belief, confidence or evidence of $40 \%$ that the 145 building is inhabitable, designated $\bar{A}$. In this case the expert does not have $100 \%$ evidence (belief or 146 confidence) of the status of the structure.

\section{Possibility/Necessity Measures}

Possibility Theory differs from Probability Theory in that it explicitly recognizes the case when 
151 evidence regarding the contrary (Dubois 2006; Dubois and Prade 1988). In Possibility Theory, to

152 characterize fully the uncertainty of $\underline{\mathrm{A}}$, uncertainty is represented by dual measures, termed possibility and 153 necessity measures (Ayyub and Klir 2006):

154

155

156

157

$$
\operatorname{Pos}_{E}\left(\{x\}=\left\{\begin{array}{l}
1 \text { when } x \in E \\
0 \text { when } x \in \bar{E}
\end{array} \quad \text { for all } x \in X\right.\right.
$$

where all alternatives in set $\mathrm{E}$ are possible, and where $\bar{E}$ is the complement of E. As shown, the Possibility measure is 1 when $x$ is within $\mathrm{E}$, and 0 when $x$ is within anything other than $\mathrm{E}$. The Necessity measure is then calculated by subtracting from 1 the possibility measure for anything other than E.

These measures are founded on the basic concepts of Possibility Theory. Possibility Theory provides a mathematical framework to represent ignorance explicitly (Ross 2010). In this context, pairs of necessity and possibility measures are linked to the mathematical framework of Evidence Theory (Dempster-Shafer Theory).

\section{Evidence Theory (Dempster-Shafer Theory) Belief/Plausibility Measures}

Evidence Theory (Dempster-Shafer Theory) is based on a measure of degree of belief, called a belief measure, $\operatorname{Bel}(\underline{\mathrm{A}})$, which expresses a degree of belief that the correct or true alternative belongs to the set $\underline{\mathrm{A}}$, from which a basic assignment or Mobius Measure, $\mathrm{m}(\mathrm{x})$, can be calculated. Mobius measures are related to the previously discussed belief and plausibility measures, and provide "an assessment of the likelihood of each set in a family of sets identified by the analyst" (Ayyub and Klir 2006). In other words, Mobius Measures are the evidence that is compiled for each event. Belief and plausibility measures are calculated as follows (Aven et al. 2014):

$$
P l(\underline{A})=\sum_{\underline{B} \cap \underline{A} \neq \varnothing} m(\underline{B})
$$


175 in which the belief in $\underline{\mathrm{A}}$ is the sum of all Mobius measures relating to $\underline{B}$ in which $\underline{B}$ is fully contained

176 within or equal to $\underline{\mathrm{A}}$ (recall that in the mathematical derivations, $\underline{\mathrm{A}}$ and $\underline{\mathrm{B}}$ are considered to be sets). The

177 plausibility measure is then the sum of all Mobius measures relating to $\underline{B}$ in which $\underline{A}$ and $\underline{B}$ have any

178 possible commonality. The plausibility measure $\mathrm{Pl}(\underline{\mathrm{A}})$ represents not only the evidence represented by the

179 belief $\operatorname{Bel}(\underline{\mathrm{A}})$, but also the evidence associated with any sets which overlap with $\underline{\mathrm{A}}$. Hence, at a minimum,

180 the plausibility will be as strong as indicated by a belief. From these equations, it is clear that the

181 relationship between plausibilities and belief measures are related through the following (Ayyub and Klir

182 2006):

183

reason, Dempster-ShaferTheory differs from classical Probability Theory in that it provides a natural

$$
\text { Ignorance }=1-[\operatorname{Bel}(\underline{\mathrm{A}})+\operatorname{Bel}(\underline{\bar{A}})]
$$

A degree of belief or evidential support of $\underline{A}, \operatorname{Bel}(\underline{A})$, does not implicate disbelief of $\underline{\bar{A}}$. For this For reference, the inverse relationship to Eq. 4 between beliefs and Mobius measures is given below (Klir 2006):

$$
m(\underline{A})=\sum_{\underline{B} \subseteq \underline{A}}(-1)^{|\underline{A}-\underline{B}|} \operatorname{Bel}(\underline{B})
$$

\section{Belief Combination using Evidence Theory (Dempster-Shafer Theory)}

Another facet of Evidence Theory is the ability to combine information from multiple sources, which can be thought of as a joint message, or a joint evidence assignment of the two pieces of evidence 
(Shafer 1987). Thus, beliefs from multiple experts are combined by first computing their Mobius measures. Combining beliefs using the Dempster-Shafer Theory (or Dempster's Rule of Combination) is well established (Klir 2006) by using Eq.10.

$$
m_{1,2}(\underline{A})=\frac{\sum_{\underline{B} \cap \underline{C}=\underline{A}} m_{1}(\underline{B}) \cdot m_{2}(\underline{C})}{1-c}
$$

Where the denominator is calculated using Eq.11 below:

$$
c=\sum_{\underline{B} \cap \underline{C}=\emptyset} m_{1}(\underline{B}) \cdot m_{2}(\underline{C})
$$

The numerator is determined by multiplying the belief in every event or event combination in which the only commonality is the event in question. Every combination is summed. The denominator is then determined by multiplying the belief in every event or event combination that has nothing in common, and summing the results. The results vary based on the evidence provided for the other events, as well as the amount of belief in a combination of events, as opposed to single events (i.e., the belief in the occurrence of either $\underline{\mathrm{A}}$ and/or $\underline{\mathrm{B}}$ versus the belief in $\underline{\mathrm{A}}$ or $\underline{\mathrm{B}}$ singly). Therefore, probability theory can be considered as a special case of evidence theory, with the former restricted to single events and the associated derivation of unions and intersections (Ayyub, 2001).

The concepts of combining judgment from multiple experts in a mathematically-founded framework could be very powerful in combining engineering judgment with quantitatively- and qualitatively-based risk calculations. Field judgment in damage assessment and building vulnerability has great potential to take advantage of Evidence Theory combinations of belief and necessity, as is demonstrated by Ballent et al. (2018).

\section{Literature Review Conclusion}

As shown in the referenced literature, evidence theory expands traditional probability assessment by providing for two measures associated with events: the belief and the plausibility. By using a model that accounts for the uncertainty in the data, it is possible to achieve a more robust output. While the work above reinforces the idea that uncertainty is, perhaps, not being given the appropriate consideration in risk 
assessment, the alternatives to frequentist probability need now to be evaluated in authentic scenarios.

224 Thus, this work aims to analyze Dempster Shafer Theory and its application to civil engineering to

225 determine how such uncertainties can be acknowledged.

226

227

228

\section{Exploration of Dempster-Shafer Theory for Risk Assessment of Natural hazards}

Since its origination, Dempster-Shafer Theory has undergone a fairly small amount of exploration.

Much of the available literature provides only Equations 10 and 11, along with a short example using two or three power sets. As a main goal of this research was to determine how Dempster-Shafer Theory could practically be put to use in civil engineering applications for natural hazard risk assessment in evaluating sustainable and resilient infrastructure, a comprehensive exploration was performed to determine trends and behavior. These include combining:

$>$ different sets of beliefs

$>$ identical sets of beliefs

$>$ power sets with missing information

$>$ power sets with extra confidence in combined events

$>$ power sets with varying amounts of ignorance

power sets with strongly conflicting beliefs.

In order to give the reader an idea of the variations that will be explored, Figure 1 provides a simple flow diagram of these explorations. In these explorations, the symbols A, B and C will now be used to represent events (singletons, rather than sets), and $\mathrm{AB}, \mathrm{AC}, \mathrm{BC}$ and $\mathrm{ABC}$ as unions or combined events. It is important to caution the reader that the standard usage in evidence theory is to designate, e.g., $\mathrm{AB}$ as the union of beliefs, rather than employing the common probability symbol AUB. This abbreviated form is consistent with the concept of power sets always being composed of unions. All examples throughout the paper will be based on these three singleton events and their combinations. It is noted that all of these 
variations begin with beliefs, rather than monotone measures. Asking experts to quantify their beliefs in various outcomes is the most natural way to solicit information from them.

\section{Combining Different Beliefs - A Practical Example}

Dempster-Shafter (Evidence) Theory can be used to combine the beliefs of multiple experts to achieve a combined Mobius measure, which can then be used to calculate a combined belief value. When experts provide a full power set of information (their belief for any single event and any combination of events), then the belief in single events along with any extra belief they have in combinations of events is redistributed to the event with the most information.

As an example, five different individual expert opinions are shown in Table 1, along with their combined belief. It is important to note that the terms A, B, etc. in Table 1 represent events, as differentiated from $\underline{\mathrm{A}}$ and $\underline{\mathrm{B}}$, where the underscore indicates sets. The information in Table 1 comes from a post-seismic damage assessment of the 2010 Port-au-Prince, Haiti earthquake. Aerial images were used to ask experts to assess their degree of belief in the degree of damage shown in the photograph (there were many photographs analyzed, and just one is presented here). The events were defined as shown below:
A $\quad 0 \%-33 \%$ damage
B $\quad 34 \%-66 \%$ damage
C $\quad 67 \%-100 \%$ damage
$\mathrm{AB} \quad 0 \%-66 \%$ damage
AC $\quad 0 \%-33 \%$ damage or $67 \%-100 \%$ damage
BC $\quad 34 \%-100 \%$ damage
$\mathrm{ABC} \quad 0 \%-100 \%$ damage 
272 then, are equal to 1 because that is the belief that either A, B, or C will happen. As these are the only

273 options, at least one of them must occur. The belief values are shown for each expert, denoted as "b1" for

274 expert 1, and so on. Recall that the belief value is what is provided by the expert.

275 Table 1 presents the results from five experts. In each case, the beliefs were converted into

276 monotone measures using Eq. 9, and then these measures were combined using Eqs. 10 and 11. The

277 resulting combined monotone measures were then converted back into beliefs using Eq. 4. It can be seen

278 that while combined ranges $\mathrm{AB}$ and $\mathrm{BC}$ had slightly higher results, the $\mathrm{A}$ range $(0 \%-33 \%)$ had almost as

279 much belief, reaching about 95\%. This is consistent with the actual ground-verified damage of $0 \%-33 \%$.

280 This strong combined belief in event A occurs even though the average of the five experts for event $\mathrm{A}$ is 281 only 0.248 . 
By looking at the initial beliefs for each event and then the combined belief, it is evident that the

combined belief is dependent on several factors. It is important to look at the starting individual event values, but also the amount of extra information provided with the belief in combined events. The added certainty that one may have in a combination of events without having to associate it with any individual event is filtered back to the single events when beliefs are combined. For example, examine event A. Each expert provides a belief value for the single event of A, but their beliefs for any combined event involving $\mathrm{A}$ ("AB" or "AC") is almost always higher than simply the combination of those individual event beliefs. When these beliefs are combined, a high combined belief for the single event of A is produced. Through this process, experts are allowed to express uncertainty or ignorance on their belief of any single event without ignoring evidence they may have on combined events.

\section{Combining Identical Beliefs}

An interesting result of this calculation occurs when evidence from people with identical beliefs is combined. Rather than resulting in the combined expert belief equaling the identical individual beliefs, the combined belief is redistributed based on the strength of the original beliefs. The belief is distributed with priority on the events with the strongest starting belief and with the most amount of extra certainty from joint events. To illustrate this point, one set of beliefs was chosen and then duplicated to calculate the combined belief as if several experts had the exact same belief. Again, this example is based on three singleton events and their combinations.

This examination was performed with the use of a computer program written in Matlab that combines expert beliefs using Dempster-Shafter Theory based on the number of experts. It should be noted that the program written for this purpose allows the combination of up to five experts. However, the results are continuous in that combining two sets of two experts with any pairing will yield the same result as combining 4 experts of the same beliefs. At this point it is also important to note that combining one set of two experts with one set of three experts does not yield the same result as combining five experts, as this weights the beliefs differently. This was verified via preliminary testing on the program. 
Using this, up to 20 experts were combined to analyze the trends. There are gaps at 7,11,13,14, 17, and

19 experts, as these numbers are not divisible by the available 1-5 expert combinations. It is worth noting that with just the equation for combining two experts, applied recursively, there would be a gap anytime the number of experts, $n$, is not equal to $2^{\mathrm{n}}$. The results of this exploration are shown below in Table 2 alongside the results of averaging the same set of beliefs using Probability Theory for comparison. The identical initial beliefs of the individual experts are shown in Table 2 with the combined belief of 20 313 experts using both Evidence Theory and Probability Theory.

Although the original power set of beliefs provides extra confidence in every double event 315 beyond the confidence of any single event, the A event acquires the most combined evidence with every added expert. While A starts out with the highest single event belief in the power set in Table 2, it also 317 gains the most from the extra belief associated with the combined events of AB and AC. Since every expert has more overall belief in event $\mathrm{A}$ than he or she does in $\mathrm{B}$ or $\mathrm{C}$, the evidence for $\mathrm{A}$ will accumulate with each added expert, therefore the combined evidence value for A will continue to approach 1 and all other values will approach 0. The belief plot shown in Figure 2 reflects this compiled 321 evidence in that the belief in any event involving A will approach 1, while any event absent of A will 322 approach 0 . This is expected, as the more evidence there is for A, the higher the belief is that any event 323 where $\mathrm{A}$ is an option will occur. The trends shown in Figure 2 are interesting to examine. With each 324 additional expert, the dominant beliefs for events $\mathrm{A}, \mathrm{AB}$ and $\mathrm{AC}$ show steady increases, tending toward 325 their asymptotic values of 1 . The events $\mathrm{C}$ and $\mathrm{BC}$, however, first show modest increases due to the 326 strong shared initial belief in $\mathrm{AC}$ ( $\mathrm{C}$ increases from 0.05 to about 0.20 while $\mathrm{BC}$ increases from 0.10 to 327 slightly above 0.2 ), but then with more experts tend to decrease toward 0 as all of the belief is transferred 328 to the events involving A, which is converging to 1.

The implication of this aspect of Evidence Theory is very important. With probability theory, any number of experts all expressing for instance $15 \%$ belief in event A would result in a group belief of $15 \%$, 331 independent of any weighting scheme; perhaps with some expressed additional confidence in this result. 
But with EvidenceTheory, the more experts who express $15 \%$ belief in event A, the more total belief there is for event $\mathrm{A}$.

In comparing Evidence Theory to Probability Theory, note that as each expert provides more or less evidence (or their belief) of an event, the combined belief increases or decreases support for one event, rather than averaging each added belief. These results challenge the frequentist probability ideology that the most common output is the correct output. While it might seem logical that the group belief be identical to the comprising individual beliefs, Evidence Theory views the beliefs as evidence for or against each event. This evidence is then compiled for each event with each new expert's belief, producing a joint belief.

\section{Mathematical Convergence for Identical Experts}

From the trends shown in Figure 2, it is interesting to determine when the beliefs converge to a particular event, a result that has heretofore not been derived as far as the authors can determine. The smooth curves derived for Figure 2 indicate that the mathematical derivation for $2^{\mathrm{n}}$ experts will characterize this behavior for a general number of experts. In order to keep the derivation manageable, and to obtain results that can easily be interpreted, the case of just two singleton events, A and B, will be considered. The derivation is general, but the inclusion of more events would make it difficult to get a physical feel for the solution. The combined monotone measure is given by Eqs. 10 and 11, where the events are now $\mathrm{A}, \mathrm{B}$ and $\mathrm{A} \cup \mathrm{B}$ (again, recall that in those equations $\underline{\mathrm{A}}$ and $\underline{\mathrm{B}}$ represent sets for each expert, whereas for this derivation the notation will be that each set consists of three possible events, with those events being designated A, B and AUB). Because the Mobius measures must sum to 1, the following relationship exists:

$$
m_{i}(A \cup B)=1-m_{i}(A)-m_{i}(B)
$$

In which $i=$ the indicator for expert $\mathrm{i}$. 
35811 give the following results for the combined Mobius measure for event A:

$$
m_{1,2}(A)=\frac{\left[1-m_{1}(B)\right]^{2}}{1-2 m_{1}(A) m_{1}(B)}
$$

361 In which it is recalled that the $m_{i}$ values are identical for experts 1 and 2 (and the double subscript on the

362 left side indicates that the result is for the first two experts combined). Because groups of two experts can continue to be combined for any number of experts satisfying $2^{\mathrm{n}}$, the above equation can be recast in a recursive form:

$$
m_{j+1}(A)=\frac{\left[1-m_{j}(B)\right]^{2}}{1-2 m_{j}(A) m_{j}(B)}
$$

367 In which the single subscript now represents the combined Mobius measure at the end of the $j^{\text {th }}$ and $(j+1)^{s t}$

368 iteration (recall that $j=1$ would represent combining two experts, $j=2$ would be combining four experts, $369 j=3$ would be eight experts, and so on).

371 Of interest is the convergent behavior of Eq. (14) as $\mathrm{j} \rightarrow \infty$. This is determined by looking at the ratio $372 m_{\mathrm{j}+1}(\mathrm{~A}) / m_{\mathrm{j}}(\mathrm{A})$. When this ratio is less than one, then the quantity $m_{\mathrm{j}+1}(\mathrm{~A})$ will tend to zero as $\mathrm{j}$ increases, 373 and all the belief will be assigned to event B. The determining ratio of unity occurs when

$$
\left[1-m_{j}(B)\right]^{2}=m_{j(A)}\left\{1-2 m_{j}(A) m_{j}(B)\right\}
$$

376 Solving this equation gives the value of $m_{j}(\mathrm{~B})$ in terms of $m_{j}(\mathrm{~A})$ for which the series converges to $m_{j}(\mathrm{~A})$

$377 \rightarrow 0$. Of interest is the initial value of $m_{l}(\mathrm{~B})$. This is given by the following quadratic equation:

$$
m_{1}^{2}(B)+\left[2 m_{1}^{2}(A)-2\right] m_{1}(B)+\left[1-m_{1}(A)\right]=0
$$


For which the quadratic solution is,

$$
m_{1}(B)=1-m_{1}^{2}(A) \mp \sqrt{m_{1}(A)\left[1-2 m_{1}(A)+m_{1}^{3}(A)\right.}
$$

Table 3 summarizes the minimum values of $m_{1}(\mathrm{~B})$ required for specified values of $m_{1}(\mathrm{~A})$ for the combined Mobius measures to converge to all belief in event B. Formally, $m_{1}(\mathrm{~B})$ must be strictly greater than the values in the table for the belief to converge completely to event $\mathrm{B}$. The results are somewhat surprising since the Mobius measures for B must be quite large for small values of evidence for A in order to overcome the values for the combined event $A \cup B$.

\section{Dealing with "Missing Information"}

The reason to consider how missing information is treated is that asking an expert for his or her belief in any combination of events might not be practical. For example, an expert is assessing a structure and has the following options: $\mathrm{A}$ is light damage, $\mathrm{B}$ is moderate damage, and $\mathrm{C}$ is extreme damage. Asking for one's belief in any possible combination of these options, such as one's belief that the structure has either light or extreme damage but not moderate damage (designated AUC, or simply AC) does not make sense. This leaves the question of how to fill in the missing information of one's belief in "A and/or C". Several ways of handling this missing information were considered. A sample data set of five different experts' beliefs was used, with the value of the belief in A or C being calculated differently each time. These beliefs were then combined to see how the different treatments of AC affected the trend of combined Mobius measures and beliefs.

The initial evaluation was done with full power sets to provide combined values for comparison. That is, the belief values of AC were provided by the experts. For this particular data set, every expert's belief in $\mathrm{AC}$ is more than just the sum of the beliefs in A and $\mathrm{C}$, signifying that each expert has increased confidence in either one of those events occurring. 
The first attempt at filling the missing $\mathrm{AC}$ information was to set the combined belief of $\mathrm{A}$ and $\mathrm{C}$ equal to the sum of the individual beliefs in A and C. Using the idea of common probability, the belief in

406 A or C should simply be the sum of the beliefs in A and C. However, since Dempster-Shafer Theory

407 allows for extra belief in combined events rather than just the combination of single events, one needs to

408 consider that the combined belief in $\mathrm{A}$ and $\mathrm{C}$ might be more than just the combined belief in the

409 individual events. If extra information is provided for $\mathrm{AB}$ and $\mathrm{BC}$, but $\mathrm{AC}$ is simply the sum of the

410 individual beliefs in $\mathrm{A}$ and $\mathrm{C}$, the combined beliefs for $\mathrm{A}$ and $\mathrm{C}$ will be negatively affected even if that is

411 not the true belief of the experts. Since the provided beliefs for AC in the original power set did have

412 extra information, the combined Mobius measures and beliefs were strongly influenced by the lack of 413 extra information in this treatment.

Therefore, the combined belief of $\mathrm{AC}$ was set equal to the belief in $\mathrm{A}, \mathrm{B}$, or $\mathrm{C}$ minus the belief of

415 B alone (theoretically leaving behind the belief of AC). Again, this follows the general rule of probability

416 in that one's beliefs must add to 1 . Therefore, the belief in 2 of 3 events should be 1 minus the belief of

417 the third event. Since Dempster-Shafer Theory allows ignorance on the part of the single events and does

418 not require these beliefs to sum to 1 (but can be no larger than 1), the calculated values of the belief in AC

419 turned out much higher than the expert-provided beliefs. This led to overly inflated values of combined

420 Mobius measures and beliefs.

421 Finally, a value of the belief in $\mathrm{AC}$ was based on the provided individual values of A and $\mathrm{C}$ while 422 also taking into account the provided extra information in $\mathrm{AB}$ and $\mathrm{BC}$. The belief value of $\mathrm{AC}$ was

423 calculated by summing the individual $\mathrm{A}$ and $\mathrm{C}$ belief values, then adding half of the extra belief assigned

424 to $\mathrm{AB}$ and $\mathrm{BC}$, with the assumption that half of the extra belief for $\mathrm{AB}$ was for $\mathrm{A}$, and half of the extra

425 belief for $\mathrm{BC}$ was for C. This is shown below in Eq. 18.

$426 \operatorname{Bel}(A \cup C)=\operatorname{Bel}(A)+\operatorname{Bel}(C)+\frac{[\operatorname{Bel}(A \cup B)-\operatorname{Bel}(A)-\operatorname{Bel}(B)]+[\operatorname{Bel}(B \cup C)-\operatorname{Bel}(B)-B e l(C)]}{2}$ 
427 While the true amount of expert belief associated with the individual events is dependent on each unique

428 assessment, this method produced combined Mobius measures and beliefs that were the closest to the

429 values calculated with the full expert-provided power set. A potential problem arises when the individual

430 A and $\mathrm{C}$ beliefs combined with the calculated added information are large enough that this calculation

431 leads to an AC belief greater than 100. If this is the case, the calculated belief in AC should logically be

432 capped at 100.

433

434

435

436

437

438

439

440

441

442

443

444

445

446

447

448

449

450

451

\section{The Effects of Extra Confidence in Combined Events}

As stated, Dempster-Shafer Theory allows experts to acknowledge that they have ignorance about individual events, but be more confident in combined events. The effect of this extra confidence was examined to determine how having low individual beliefs but significant extra beliefs in combined events might weigh against having high individual beliefs with no extra confidence in combined events. Five different power sets of information with varying amounts of "extra confidence" were evaluated by repeatedly combining them, as though several experts had the same set of beliefs, to analyze trends. These power sets are shown in Table 4, based on the three singleton events and their combinations.

The first power set gives event A a starting belief of $40 \%$, and B and C comparatively low beliefs of $10 \%$. In this first set, the belief in the double events is the sum of the single events; there is no added confidence. As expected, the combined belief in $\mathrm{A}, \mathrm{AB}$, and $\mathrm{AC}$ continued to grow while the belief in events B, C, and BC trended towards 0 . The results of this test can be seen in Table 5 and Figure 3. The second power set had the same individual event beliefs, but the extra confidence in the combined event of $\mathrm{BC}$ was increased by $10 \%$ (from 0.2 to 0.3 ), as shown in the second power set in Table 4. The results are similar to the original test, suggesting that $10 \%$ increase in added belief in $\mathrm{BC}$ was not significant enough to diminish the higher starting belief in event A. The combined belief of 10 experts still strongly supported A while belief in any event or event combination not containing A trended towards zero. 
453 belief in event $\mathrm{A}$ and the belief in $\mathrm{BC}$ equal, as shown in the third power set in Table 4. However, the

454 combined belief in A still trended toward 1, while the belief in B and C briefly increased and then trended

455 back down towards 0 . This is still expected, as the beginning beliefs in $\mathrm{AB}$ and $\mathrm{AC}$ are still greater than

456 the belief in BC. However, the combined belief in A of all 10 experts was just under 0.8; a noticeable

457 decrease from the combined belief of nearly 1 in Table 5.

458 The fourth power set increased the belief in $\mathrm{BC}$ by another $10 \%$ to a total of $50 \%$, as shown by

459 the fourth power set in Table 4. While the belief in B and $\mathrm{C}$ remain at $10 \%$, the belief in $\mathrm{BC}$ now matched 460 the beliefs in $\mathrm{AB}$ and $\mathrm{AC}$. The trends were not clear after combining 10 experts with identical beliefs, so 461 the test was extended through 20 experts. The beliefs in A, B, and C all trend towards $33 \%$, with the 462 belief in double events trending towards $67 \%$. The results of this extra belief are significant because even 463 though $\mathrm{A}$ had a significantly higher starting belief than $\mathrm{B}$ and $\mathrm{C}$, and the beliefs in $\mathrm{AB}, \mathrm{AC}$, and $\mathrm{BC}$ were 464 all identical, B and C trended upwards while A trended down. This proved that this extra belief in BC is 465 the turning point in extra confidence overtaking individual starting belief. However, these results are 466 interesting because the added belief in $\mathrm{BC}$ does not necessarily give enough belief to $\mathrm{B}$ and $\mathrm{C}$ individually 467 to have this effect. Since the belief in $\mathrm{AB}$ and $\mathrm{AC}$ are solely the sum of the individual beliefs in $\mathrm{A}$ and $\mathrm{B}$, 468 and $\mathrm{A}$ and $\mathrm{C}$, respectively, it makes sense to split the extra belief in B and C and add it back to the starting 469 beliefs of B and C. Following this theory, the starting beliefs of B and C would each gain 15\%, putting 470 them both at $25 \%$ and still less than the starting belief of $\mathrm{A}$. This suggests that extra confidence in 471 combined events is handled differently, and potentially more seriously, than starting beliefs in individual 472 events.

473 The fifth power set shown in Table 4 increased the starting belief in $\mathrm{BC}$ to $60 \%$. Again, the 474 individual belief in $\mathrm{B}$ and $\mathrm{C}$ remain low, but the belief in $\mathrm{BC}$ is now higher than $\mathrm{AB}$ or $\mathrm{AC}$. This higher 475 belief in $\mathrm{BC}$ than any other event dominates, driving the combined belief in $\mathrm{A}$ toward 0 (A is the only 476 event not contained in $\mathrm{BC}$ ). The corresponding belief for $\mathrm{BC}$ trends toward 1 (since the belief in $\mathrm{ABC}$ 
must equal 1, and with no belief in $\mathrm{A}$, this is the same as the belief in $\mathrm{BC}$ ). The beliefs in $\mathrm{B}$ and $\mathrm{C}$ trended significantly upward, while the belief in $\mathrm{AB}$ and $\mathrm{AC}$ stayed at $50 \%$, evidently unaffected by the extra belief in $\mathrm{BC}$, since with an increasing number of experts that extra belief in $\mathrm{BC}$ is now being distributed to $\mathrm{B}$ and $\mathrm{C}$, as they trend toward 0.5 (joining $\mathrm{AB}$ and $\mathrm{AC}$ ). Based on the previous test results, it was expected that $\mathrm{B}$ and $\mathrm{C}$ would outweigh the belief in A with this extra belief in $\mathrm{BC}$. The results of this test can be seen in Table 6 and Figure 4.

The purpose of these explorations was to examine how Dempster-Shafer Theory handles the extra confidence that one may have in the belief of either one of two options, rather than choosing between the two. The results are interesting in that the extra confidence in combined events appears to be more heavily weighted than the belief in single events. On one hand, this seems counterintuitive since the expert was not confident enough to assign any of the extra belief to the individual events, only to the chance of their either/or occurrence. On the other hand, this makes sense in that the expert is confident enough that one of the two will occur, but might be uncertain about the split between the individual events and is uncomfortable choosing to assign more belief to either of the two.

\section{Varying Amounts of Ignorance}

A key difference between Evidence (Dempster-Shafer) Theory and Probability is that DempsterShafer Theory does not require one to assign all of his or her belief to any individual or combination of events. Ignorance is allowed and, accordingly, the sum of one's beliefs is often less than unity. The impact of the amount of ignorance one can have was explored to determine how combining experts with relatively low beliefs might differ from combining experts with higher, though proportional, beliefs. For the purposes of identifying trends, one set of beliefs was repeatedly combined until the combined expert belief no longer had any ignorance, or all $100 \%$ of the belief was accounted for among the three single events. In these examples, the singleton events $\mathrm{A}, \mathrm{B}$ and $\mathrm{C}$ will continue to be used.

The first examination uses a set of beliefs that has a large amount of ignorance. There was only $10 \%$ belief for the single events and $20 \%$ for the double events (the sum of their respective single events 
with no extra confidence). This leaves $70 \%$ of one's belief unassigned. As stated previously, the belief in

504 A, B, or C must always be $100 \%$ as there are no other events that may occur. This set of beliefs required

50520 experts to reach a point when the belief of the individual events did sum to $100 \%$ (with a belief of

$506 \sim 33 \%$ for each individual event) and there was no longer any ignorance, as probability would require. The

507 combined beliefs can be seen in Table 7.

508

These results demonstrate how partial evidence is handled with Dempster Shafer Theory. While initially, the experts have very little belief assigned to any single event, the same small amount of belief is assigned

512 for each event until the maximum amount of evidence is reached (33\% for each event if dealing with 3 events). With this set of starting beliefs, 20 experts are required to contribute beliefs in order to compile enough evidence to reach $33 \%$ for each event. While in theory an infinite number of experts would be needed to reach the asymptotic values, it is noted that with 9-10 experts the beliefs are within a relative error of $10 \%$ of their asymptotic values \{e.g., 0.30 compared to 0.33 , and 0.60 compared to 0.65 ). were required to reduce the ignorance to zero. Each individual event was assigned a belief of $20 \%$ with 519 the double events again being the sum of their respective individual events (40\%), leaving $40 \%$ of the 520 belief unassigned. This set of beliefs required 8 experts to reach a combined belief with no ignorance. component of Dempster-Shafer Theory that makes it a contending alternative to probability. The tests

527 above suggest that the ignorance provided by experts is retained in their combined belief until enough 
evidence is provided to allow otherwise. When there is substantial ignorance in the starting beliefs, many

529 experts are required to contribute their belief to reach a combined belief that no longer has ignorance. The

530 higher the starting beliefs, the lower the number of experts required to reach a full combined belief power

531 set with no ignorance. While no absolute rules were developed for the number of experts required to

532 remove ignorance completely, explorations such as these could be used to develop guidelines for

533 particular applications.

534

535

536

537

538

539

540

541

542

543

544

545

546

547

548

549

550

551

552

\section{Conflicting Expert Opinion}

Conflicting belief has been a noted weak point of Dempster-Shafer Theory (Xin et al. 2005). Ayyub and Klir (2006) offer alternative methods, replacing Eq. 10 with a term scaled to conflict or distributing the degree of conflict to the set of outcomes. The use of these alternatives may be important when there is a high degree of conflict. It has not been pursued here since the applications envisioned in natural hazard risk assessment for infrastructure should not in general lead to a high degree of conflict. Equations 10 and 11 have straightforward interpretations in terms of the inclusion of all terms with any overlap in Eq. 10 and terms with no overlap in Eq. 11. To determine how the equations outlined in this paper handle conflicting opinion, a series of basic tests was performed. In each case, the three singleton events $\mathrm{A}, \mathrm{B}$ and $\mathrm{C}$ and their combinations are considered. The first test assigned absolute belief in event $\mathrm{A}$ to one expert and absolute belief in event B to a second. The belief in combined events is the sum of the individuals. In such cases of $100 \%$ conflicting belief, this theory is not able to compute a joint belief as there is no commonality between the two experts, from which the numerator in Eq. 10 originates.

The second test assigns near absolute belief to the same events as the first, but with $1 \%$ ignorance. In this case, Dempster-Shafer Theory essentially takes the average of the provided beliefs as there is nearly no commonality between the experts, but both admit some small ignorance. Since Probability Theory would handle these beliefs in a similar method, this seems like a natural result. The results are outlined in Table 8. 
A third test was carried out by assigning three experts $50 \%$ belief in different events. Rather than

555 producing an average combined belief, the result was a 25\% joint belief in each individual event (larger

556 than the $16.67 \%$ that an average would yield) and a $50 \%$ belief in each combined event, as seen in Table

557 9. This result reflects a notable difference in how probability and Dempster-Shafer Theory deal with

558 multiple inputs and significant ignorance.

559 In order to analyze how larger quantities of conflicting beliefs are handled, one expert was

560 assigned absolute belief (100\%) in event $\mathrm{C}$, which was combined with three other experts that all had

561 strong belief (90\%) in A. This test is of significant interest because even though it seems there is more

562 overall belief in event A, the first expert's absolute belief in C allows no ignorance or commonality, and

563 therefore trumps the less-than-absolute belief that the other three experts had in event A. The resulting

564 combined belief was $100 \%$ belief in $\mathrm{C}$ and $0 \%$ in A. Again, the idea of ignorance plays a significant role

565 in Dempster-Shafer Theory that probability would ignore.

566 The implications of this complete (or near) confidence of a single expert, in this case for event $\mathrm{C}$, requires

567 comment. In a democratic process. It would be possible for a single expert to negate the opinions of other

568 experts by assigning such complete belief to the outcome preferred by this one expert. For instance, in the

569 case of the first example in this paper, one expert could "decide" how much damage is revealed from the

570 aerial photography. Such an outcome would be at least as bad as the shortcoming of probability theory of

571 ignoring the extra strength of belief from consistent experts. Further considerations of such limitations

572 deserve continued evaluation and study before one can be fully comfortable in promoting the use of

573 Dempster-Shafer theory.

574 A fourth test was performed to determine at what level of confidence expert 1's belief in event C

575 gives way to the other three experts' belief in event A. Keeping the first three experts' belief in event A at $57690 \%$, the confidence that expert 1 has in $\mathrm{C}$ was varied from $90 \%$ to $100 \%$. When expert 1 's confidence

577 drops below around 99.5\%, the combined belief shifts towards event A. Any belief above 99.5\% does not 
578 leave enough ignorance to allow for the other three expert's beliefs to influence the combined belief. This

579 last scenario demonstrates how different belief measures are from probability. Belief measures represent

580 one's confidence in a certain outcome. When this confidence is near $100 \%$, this is taken almost as fact of

581 what will happen, rather than just an estimate. The remaining ignorance is so small that other beliefs less

582 than $100 \%$ are considered negligible or not likely enough to occur.

583

584

585

586

587

588

589

590

591

592

593

594

595

596

597

598

599

600

601

602

603

\section{Summary of Program Explorations}

The series of explorations performed on the MatLab programs and the new mathematical derivation helped determine how Dempster-Shafer (Evidence) Theory behaves in specified situations and in which scenarios it might be applicable. Several key outcomes were determined throughout this exploration, many of which highlighted the contrast between this theory and basic probability. Combining expert beliefs using Evidence Theory yields a significantly different result from simple averaging; as more and more beliefs contribute, one event will eventually reach a joint belief of $100 \%$, while all other single events will have $0 \%$. Several other aspects influence the joint belief, including any amount of ignorance the experts may have (a total belief less than 100\%), how much extra belief he or she has in the joint events versus the single events, and how conflicting the contributing beliefs are. The ignorance allowed in beliefs less than $100 \%$ act as a sort of weighting measure - experts with more ignorance do not influence the joint belief as strongly as experts who assign $100 \%$ of their belief. The confidence level of the contributing experts influences how many experts are required to reach total belief in a definitive answer. For example, a small number of very confident experts with similar beliefs might have a joint belief of $100 \%$ in one event, while a larger group of less certain experts may yield a more ambivalent result. When contributing beliefs are strongly conflicting, the amount of ignorance present plays a key role. One expert who very strongly believes in event A (little to no ignorance) combined with another expert who has a moderately high belief in event B (slightly more ignorance), will yield a joint belief that strongly backs event A, even though the contributing beliefs in A and B are both high. Another key outcome of conflicting belief is that when there is no possible overlap, for example if one expert has 
605 joint belief.

Overall, these results were able to provide a general foundation for the behavior and trends of

607 Dempster Shafer Theory under several conditions. This basis allows for further real-world testing in

608 practical risk situations, such as the survey instrument used by Ballent et al. (2018).

\section{Conclusion}

A primary goal of this paper was to further understand the role of uncertainty and expert belief in assessing natural hazard risk for sustainable and resilient built environments, and to analyze potential frameworks with which this uncertainty might be captured. While a variety of frameworks have been presented in previous publications, the exploration program that was executed and analyzed in this paper offers a more comprehensive understanding of Evidence (Dempster Shafer) Theory and how such a theory would react given various realistic inputs. Evidence Theory provides significantly different results in subjective cases when compared to the frequentist alternative of probability. Such results often provide a much more definitive and involved joint belief that takes into account aspects such as what confidence levels the experts have, any extra belief there may be in a wider range of events, and how conflicting the contributing beliefs are. Using a method that contains these nuances could yield significantly different results when compared to probability. The rules of probability, namely additivity, handle a potential doubt, or lack of belief, in an event as evidence to its contrary. Many of the cases examined in this paper involved some level of ignorance, and its influence was not insignificant. By using a framework that acknowledges such a lack of belief as ignorance, rather than belief of the contrary, it is potentially possible to achieve more meaningful results. The results discussed in this paper suggest that DempsterShafer Theory is a viable, if not preferential, treatment of cases that involve uncertainty.

Dempster-Shafer Theory was put through several trials in this paper to determine general trends and behaviors, but many unknowns remain. The particular equation analyzed here showed some 
limitations, such as with strongly conflicting beliefs that have no commonality. Alternatives might provide an improvement in such situations, and should be investigated further.

The main applications of Dempster-Shafer Theory considered in this research are within the field of natural hazard assessment, but the possibilities extend far beyond that. Subjective investigations and assessments are unavoidable in many fields, as no two locations, projects, communities, and environments are exactly the same. These circumstances present the challenge of recognizing and accounting for such uncertainties. A mathematical framework such as Dempster-Shafer Theory that allows for this uncertainty has the potential to change the outcome of civil engineering decisions on a large scale. Nevertheless, such a radical change in handling expert opinion and evidence must explore unintended consequences, such as dominance by a single expert, along with the potentially significant benefits.

\section{Acknowledgments}

The authors gratefully acknowledge the support of the National Science Foundation; grant number CMMI 1552855. The opinions expressed in this paper are those of the authors, and do not necessarily reflect the views or policies of the NSF.

\section{References}

Adeli, H. (1988). "Artificial Intelligence and Expert Systems.” Expert Systems in Construction and Structural Engineering. Ed. H. Adeli. London: Chapman and Hall, 1-12.

Aven, T., Baraldi, P., Flage, R., and Zio, E. (2014). Uncertainty in Risk Assessment: The Representation and Treatment of Uncertainties by Probabilistic and Non-Probabilistic Methods. John Wiley \& Sons, Ltd, The Atrium, Southern Gate, Chichester, West Sussex, United Kingdom.

Ayyub, B. M. (1998). Uncertainty Modeling and Analysis in Civil Engineering. CRC Press LLC, Boca Raton, Florida.

Ayyub, B. M. (2001). Elicitation of Expert Opinions for Uncertainty and Risks. CRC Press LLC, Boca Raton, Florida.

Ayyub, B. M., and Klir, G. J. (2006). Uncertainty Modeling and Analysis in Engineering and the Sciences. Chapman \& Hall/CRC, Boca Raton, Florida.

Ballent, W. (2018). "Dempster-Shafer Theory Applications in Structural Damage Assessment and Social Vulnerability Ranking." Masters, University of Colorado, Boulder, Boulder, CO. 
Ballent, W., Corotis, R. B., and Torres-Machi, C. (2018). "Dempster Shafer Theory Applications in PostSeismic Structural Damage and Social Vulnerability Assessment [SUBMITTED].” Earthquake Spectra.

Beynon, M., Curry, B., and Morgan, P. (2000). "The Dempster-Shafer theory of evidence: an alternative approach to multicriteria decision modelling." The International Journal of Management Science, 28(1), 37-50.

Booker, J. M. and Ross, T. J. (2011). "An evolution of uncertainty assessment and quantification." Scientia Iranica 18(3), 669-676.

Cooke, R. M. (1991). Experts in Uncertainty: Opinion and Subjective Probability in Science. Oxford University Press, Oxford, United Kingdom.

Corotis, R. B. (2015). "An Overview of Uncertainty Concepts Related to Mechanical and Civil Engineering." ASCE-ASME Journal of Risk and Uncertainty in Engineering Systems, Part B: Mechanical Engineering, 1(4), 12.

Dubois, D. (2006). "Possibility Theory and Statistical Reasoning." Computational Statistics \& Data Analysis, 51(1), 47-69.

Dubois, D., and Prade, H. (1988). Possibility Theory: An Approach to Computerized Processing of Uncertainty. Springer US, New York.

Klir, G. J. (2006). Uncertainty and Information Foundations of Generalized Information Theory. Wiley IEEE, John Wiley \& Sons, Inc, Hoboken, New Jersey.

Klir, G. J., and Smith, R. M. (2001). "On measuring uncertainty and uncertainty-based information: Recent developments." Annals of Mathematics and Artificial Intelligence, 32(1-4), 5-33.

Melchers, R. E. (1999). Structural Reliability Analysis and Prediction. John Wiley \& Sons, Ltd, Chichester, West Sussex, England.

Ross, T. J. (2010). Fuzzy Logic with Engineering Applications. John Wiley \& Sons, West Sussex, England.

Shafer, G. (1976). A Mathematical Theory of Evidence. Princeton University Press, Princeton, New Jersey.

Shafer, G. (1987). "Belief Functions and Possibility Measures." Analysis of Fuzzy Information, J. C. Bezdek, ed., CRC Press, Boca Raton, Florida, 51-84.

Vick, S. G. (2002). Degrees of Belief Subjective Probability and Engineering Judgment. ASCE Press, Reston, Virginia.

Walley, P. (1991). Statistical Reasoning with Imprecise Probabilities. Monographs on Statistics \& Applied Probability, Chapman \& Hall/CRC.

Wang, Z., and Klir, G. J. (2009). Generalized Measure Theory. IFSR International Series on Systems Science and Engineering, Springer Science + Business Media, LLC, New York, NY.

Xin, G., Xiao, Y., and You, H. (2005). "An Improved Dempster-Shafer Algorithm for Resolving the Conflicting Evidences." International Journal of Information Technology, 11, 68-75.

Yager, R. R., and Liu, L. (2008). Classic Works of the Dempster-Shafer Theory of Belief Functions. Studies in Fuzziness and Soft Computing, (J. Kacprzyk, ed.), Springer, Heidelberg. 
705 Fig. 1. Flow Diagram of Explorations for Number of Experts, Extra Belief and Confidence 706 Fig. 2. Belief of Combined Experts with Identical Beliefs

707 Fig. 3. Joint Expert Beliefs with No Extra Confidence in Combined Events

708 Fig. 4. Joint Belief Power Sets with $40 \%$ Extra Confidence in Combined Events 709

710 
Table 1. Combined Belief of Experts with Different Individual Beliefs

\begin{tabular}{ccccccc}
\hline Event & $\boldsymbol{b} 1$ & $\boldsymbol{b} 2$ & $\boldsymbol{b 3}$ & $\boldsymbol{b} 4$ & $\boldsymbol{b 5}$ & Combined $\boldsymbol{b}$ \\
\hline $\mathbf{A}$ & 0.58 & 0.25 & 0.059 & 0.25 & 0.1 & 0.953 \\
\hline $\mathbf{B}$ & 0.17 & 0.31 & 0.24 & 0.13 & 0.2 & 0.023 \\
\hline $\mathbf{C}$ & 0.042 & 0 & 0 & 0 & 0.033 & 0.01 \\
\hline $\mathbf{A B}$ & 0.75 & 0.63 & 0.59 & 0.63 & 0.47 & 0.976 \\
\hline $\mathbf{A C}$ & 0.83 & 0.63 & 0.47 & 0.63 & 0.53 & 0.983 \\
\hline $\mathbf{B C}$ & 0.21 & 0.31 & 0.24 & 0.13 & 0.33 & 0.034 \\
\hline $\mathbf{A B C}$ & 1 & 1 & 1 & 1 & 1 & 1 \\
\hline \multicolumn{7}{c}{ Note: b is belief }
\end{tabular}

713

Note: $b$ is belief

714

715 
720

721

722

723
Table 2. Combining Identical Expert Beliefs Using Dempster-Shafer Theory and Probability Theory

\begin{tabular}{|c|c|c|c|c|c|c|}
\hline \multirow[t]{3}{*}{ Event } & \multirow{2}{*}{\multicolumn{2}{|c|}{$\begin{array}{l}\text { Initial Beliefs } \\
\text { (1 expert) }\end{array}$}} & \multicolumn{4}{|c|}{$\begin{array}{l}\text { Combined Beliefs } \\
\text { (20 experts) }\end{array}$} \\
\hline & & & \multicolumn{2}{|c|}{ Probability } & \multicolumn{2}{|c|}{ Evidence } \\
\hline & bel & $m$ & $m$ & bel & $m$ & bel \\
\hline $\mathbf{A}$ & 0.15 & 0.15 & 0.15 & 0.15 & 0.92 & 0.92 \\
\hline B & 0.00 & 0.00 & 0.00 & 0.00 & 0.00 & 0.00 \\
\hline $\mathbf{C}$ & 0.05 & 0.05 & 0.05 & 0.05 & 0.08 & 0.08 \\
\hline $\mathbf{A B}$ & 0.20 & 0.15 & 0.05 & 0.20 & 0.00 & 0.92 \\
\hline $\mathbf{A C}$ & 0.40 & 0.20 & 0.20 & 0.40 & 0.01 & 1.00 \\
\hline BC & 0.10 & 0.05 & 0.05 & 0.10 & 0.00 & 0.08 \\
\hline $\mathbf{A B C}$ & 1.00 & 0.50 & 0.50 & 1.00 & 0.00 & 1.00 \\
\hline
\end{tabular}


Table 3. Minimum Evidence for Event B per Expert for Convergence to Event B

725

\begin{tabular}{ccc}
\hline $\boldsymbol{m}_{\mathbf{1}}(\mathbf{A})$ & $\boldsymbol{m}_{\mathbf{1}}(\mathbf{B})$ & $\boldsymbol{m}_{\mathbf{1}}(\mathbf{A} \cup \boldsymbol{B})$ \\
\hline 0.1 & 0.707 & 0.193 \\
\hline 0.2 & 0.611 & 0.189 \\
\hline 0.3 & 0.592 & 0.108 \\
\hline 0.4 & 0.515 & 0.089 \\
\hline 0.5 & 0.5 & 0 \\
\hline
\end{tabular}

726

727

728

729

730

731 


\begin{tabular}{cccccc}
\hline Event & Set 1 & Set 2 & Set 3 & Set 4 & Set 5 \\
\hline A & 0.4 & 0.4 & 0.4 & 0.4 & 0.4 \\
\hline B & 0.1 & 0.1 & 0.1 & 0.1 & 0.1 \\
\hline C & 0.1 & 0.1 & 0.1 & 0.1 & 0.1 \\
\hline AB & 0.5 & 0.5 & 0.5 & 0.5 & 0.5 \\
\hline AC & 0.5 & 0.5 & 0.5 & 0.5 & 0.5 \\
\hline BC & 0.2 & 0.3 & 0.4 & 0.5 & 0.6 \\
\hline ABC & 1 & 1 & 1 & 1 & 1 \\
\hline
\end{tabular}

734

735

736 
Table 5. Combined Expert Beliefs with No Extra Confidence in Combined Events

\begin{tabular}{cccccccccc}
\hline $\begin{array}{c}\text { \# of } \\
\text { Experts }\end{array}$ & $\mathbf{1}$ & $\mathbf{2}$ & $\mathbf{3}$ & $\mathbf{4}$ & $\mathbf{5}$ & $\mathbf{6}$ & $\mathbf{8}$ & $\mathbf{9}$ & $\mathbf{1 0}$ \\
\hline $\mathbf{A}$ & 0.4 & 0.59 & 0.71 & 0.79 & 0.86 & 0.91 & 0.96 & 0.97 & 0.98 \\
\hline $\mathbf{B}$ & 0.1 & 0.11 & 0.10 & 0.08 & 0.06 & 0.04 & 0.02 & 0.01 & 0.01 \\
\hline $\mathbf{C}$ & 0.1 & 0.11 & 0.10 & 0.08 & 0.06 & 0.04 & 0.02 & 0.01 & 0.01 \\
\hline $\mathbf{A B}$ & 0.5 & 0.70 & 0.80 & 0.87 & 0.92 & 0.95 & 0.98 & 0.99 & 0.99 \\
\hline $\mathbf{A C}$ & 0.5 & 0.70 & 0.80 & 0.87 & 0.92 & 0.95 & 0.98 & 0.99 & 0.99 \\
\hline BC & 0.2 & 0.22 & 0.19 & 0.15 & 0.11 & 0.08 & 0.04 & 0.03 & 0.02 \\
\hline $\mathbf{A B C}$ & 1 & 1 & 1 & 1 & 1 & 1 & 1 & 1 & 1 \\
\hline
\end{tabular}

739

740

741

742 
Table 6. Combined Belief Power Sets with 40\% Extra Confidence in Combined Events

744

\begin{tabular}{cccccccccc}
\hline $\begin{array}{c}\text { \# of } \\
\text { Experts }\end{array}$ & $\mathbf{1}$ & $\mathbf{2}$ & $\mathbf{3}$ & $\mathbf{4}$ & $\mathbf{5}$ & $\mathbf{6}$ & $\boldsymbol{8}$ & $\mathbf{9}$ & $\mathbf{1 0}$ \\
\hline $\mathbf{A}$ & 0.4 & 0.32 & 0.26 & 0.21 & 0.16 & 0.13 & 0.08 & 0.07 & 0.05 \\
\hline $\mathbf{B}$ & 0.1 & 0.18 & 0.24 & 0.30 & 0.34 & 0.37 & 0.42 & 0.43 & 0.45 \\
\hline $\mathbf{C}$ & 0.1 & 0.18 & 0.24 & 0.30 & 0.34 & 0.37 & 0.42 & 0.43 & 0.45 \\
\hline $\mathbf{A B}$ & 0.5 & 0.50 & 0.50 & 0.50 & 0.50 & 0.50 & 0.50 & 0.50 & 0.50 \\
\hline $\mathbf{A C}$ & 0.5 & 0.50 & 0.50 & 0.50 & 0.50 & 0.50 & 0.50 & 0.50 & 0.50 \\
\hline $\mathbf{B C}$ & 0.6 & 0.68 & 0.74 & 0.80 & 0.84 & 0.87 & 0.92 & 0.93 & 0.95 \\
\hline $\mathbf{A B C}$ & 1 & 1 & 1 & 1 & 1 & 1 & 1 & 1 & 1 \\
\hline
\end{tabular}

745

746 
Table 7. Combined Experts Belief Power Sets with Ignorance of 70\%

\begin{tabular}{ccccccccccccccc}
\hline $\begin{array}{c}\text { \# of } \\
\text { Experts }\end{array}$ & $\mathbf{1}$ & $\mathbf{2}$ & $\mathbf{3}$ & $\mathbf{4}$ & $\mathbf{5}$ & $\mathbf{6}$ & $\mathbf{8}$ & $\mathbf{9}$ & $\mathbf{1 0}$ & $\mathbf{1 2}$ & $\mathbf{1 5}$ & $\mathbf{1 6}$ & $\mathbf{1 8}$ & $\mathbf{2 0}$ \\
\hline $\mathbf{A}$ & 0.1 & 0.16 & 0.20 & 0.23 & 0.25 & 0.26 & 0.28 & 0.29 & 0.30 & 0.31 & 0.32 & 0.32 & 0.32 & 0.33 \\
\hline $\mathbf{B}$ & 0.1 & 0.16 & 0.20 & 0.23 & 0.25 & 0.26 & 0.28 & 0.29 & 0.30 & 0.31 & 0.32 & 0.32 & 0.32 & 0.33 \\
\hline $\mathbf{C}$ & 0.1 & 0.16 & 0.20 & 0.23 & 0.25 & 0.26 & 0.28 & 0.29 & 0.30 & 0.31 & 0.32 & 0.32 & 0.32 & 0.33 \\
\hline $\mathbf{A B}$ & 0.2 & 0.32 & 0.40 & 0.45 & 0.49 & 0.52 & 0.57 & 0.58 & 0.60 & 0.61 & 0.63 & 0.63 & 0.65 & 0.65 \\
\hline $\mathbf{A C}$ & 0.2 & 0.32 & 0.40 & 0.45 & 0.49 & 0.52 & 0.57 & 0.58 & 0.60 & 0.61 & 0.63 & 0.63 & 0.65 & 0.65 \\
\hline $\mathbf{B C}$ & 0.2 & 0.32 & 0.40 & 0.45 & 0.49 & 0.52 & 0.57 & 0.58 & 0.60 & 0.61 & 0.63 & 0.63 & 0.65 & 0.65 \\
\hline $\mathbf{A B C}$ & 1 & 1 & 1 & 1 & 1 & 1 & 1 & 1 & 1 & 1 & 1 & 1 & 1 & 1 \\
\hline
\end{tabular}

748

749

750

751 
Table 8. Conflicting Expert Belief and 1\% Ignorance in Combined Beliefs

753

\begin{tabular}{cccc}
\hline Event & $\begin{array}{c}\text { Expert 1 } \\
\text { Belief }\end{array}$ & $\begin{array}{c}\text { Expert 2 } \\
\text { Belief }\end{array}$ & $\begin{array}{c}\text { Combined } \\
\text { Belief }\end{array}$ \\
\hline $\boldsymbol{A}$ & $99.0 \%$ & $0.0 \%$ & $49.8 \%$ \\
\hline $\boldsymbol{B}$ & $0.0 \%$ & $99.0 \%$ & $49.8 \%$ \\
\hline $\boldsymbol{C}$ & $0.0 \%$ & $0.0 \%$ & $0.0 \%$ \\
\hline $\boldsymbol{A} \boldsymbol{B}$ & $99.0 \%$ & $99.0 \%$ & $99.5 \%$ \\
\hline $\boldsymbol{A} \boldsymbol{C}$ & $99.0 \%$ & $0.0 \%$ & $49.8 \%$ \\
\hline $\boldsymbol{B} \boldsymbol{C}$ & $0.0 \%$ & $99.0 \%$ & $49.8 \%$ \\
\hline $\boldsymbol{A} \boldsymbol{B} \boldsymbol{C}$ & $100.0 \%$ & $100.0 \%$ & $100.0 \%$ \\
\hline
\end{tabular}

754

755

756

757 
Table 9. Conflicting Expert Belief and 50\% Ignorance in Combined Beliefs

\begin{tabular}{ccccc}
\hline Event & $\begin{array}{c}\text { Expert 1 } \\
\text { Belief }\end{array}$ & $\begin{array}{c}\text { Expert 2 } \\
\text { Belief }\end{array}$ & $\begin{array}{c}\text { Expert 3 } \\
\text { Belief }\end{array}$ & $\begin{array}{c}\text { Combined } \\
\text { Belief }\end{array}$ \\
\hline $\boldsymbol{A}$ & $50 \%$ & $0 \%$ & $0 \%$ & $25 \%$ \\
\hline $\boldsymbol{B}$ & $0 \%$ & $50 \%$ & $0 \%$ & $25 \%$ \\
\hline $\boldsymbol{C}$ & $0 \%$ & $0 \%$ & $50 \%$ & $25 \%$ \\
\hline $\boldsymbol{A} \boldsymbol{B}$ & $50 \%$ & $50 \%$ & $0 \%$ & $50 \%$ \\
\hline $\boldsymbol{A} \boldsymbol{C}$ & $50 \%$ & $0 \%$ & $50 \%$ & $50 \%$ \\
\hline $\boldsymbol{B} \boldsymbol{C}$ & $0 \%$ & $50 \%$ & $50 \%$ & $50 \%$ \\
\hline $\boldsymbol{A} \boldsymbol{B} \boldsymbol{C}$ & $100 \%$ & $100 \%$ & $100 \%$ & $100 \%$ \\
\hline
\end{tabular}

760

761 

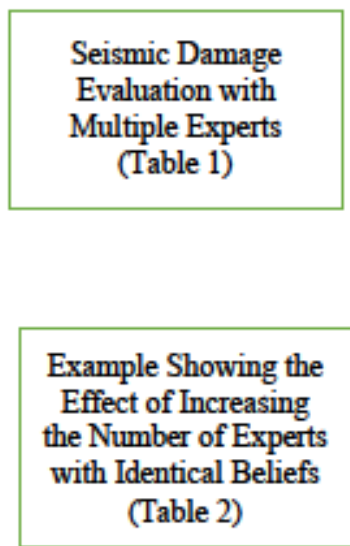

Effect of Increasing the Number of Experts with Identical Beliefs

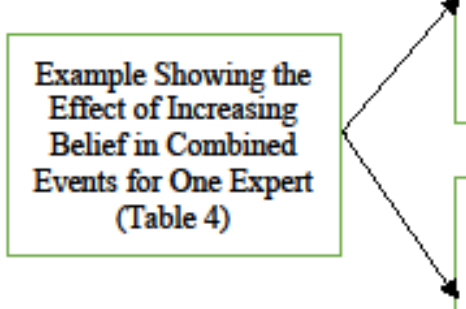

(No extra BC belief) (Table 5)

Effect of Increasing the Number of Experts with Identical Beliefs (High extra BC belief)

\section{Example Showing Decreasing Ignorance with Increased Number of Identical Experts (Table 7)} (Table 6)

\begin{tabular}{|c|c|c|}
\hline $\begin{array}{c}\text { Example Showing } \\
\text { Conflicting Beliefs } \\
\text { among Experts } \\
\text { (Table 8) }\end{array}$ & Increasing & $\begin{array}{c}\text { Example Showing } \\
\text { Conflicting Beliefs } \\
\text { among Experts } \\
\text { (Table 9) }\end{array}$ \\
\cline { 3 - 3 } & Ignorance & \begin{tabular}{c} 
(T) \\
\hline
\end{tabular}
\end{tabular}




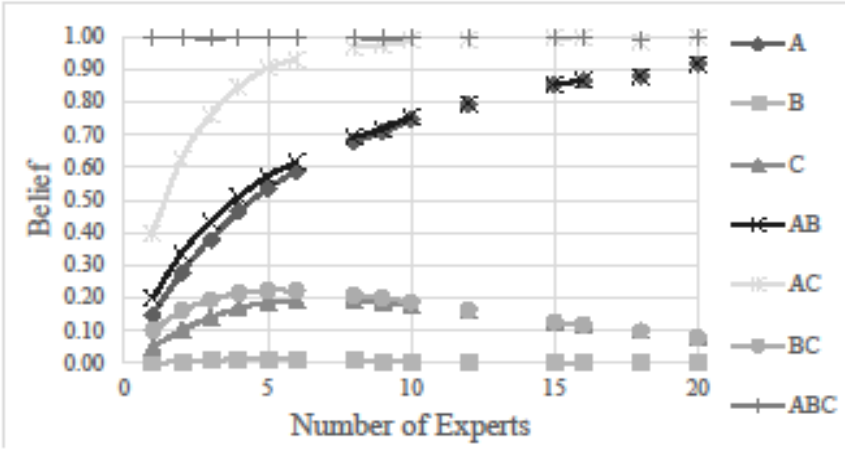

765 Fig. 2. Belief of Combined Experts with Identical Beliefs

766 


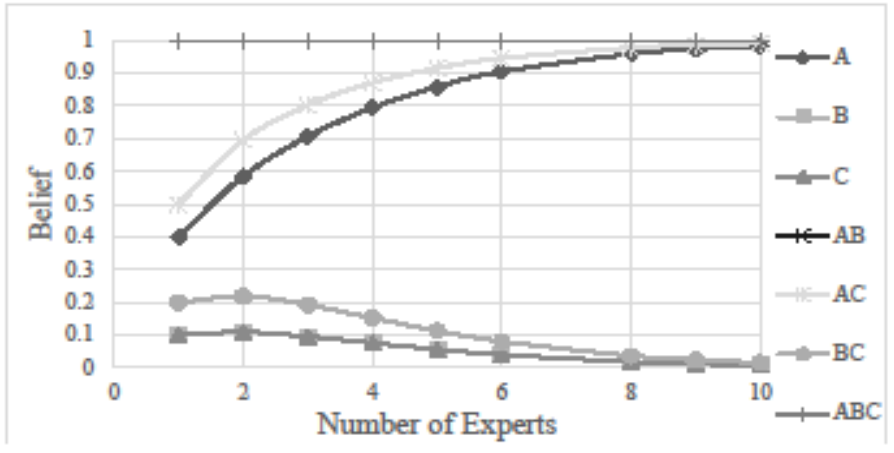

768 Fig. 3. Joint Expert Beliefs with No Extra Confidence in Combined Events 769 


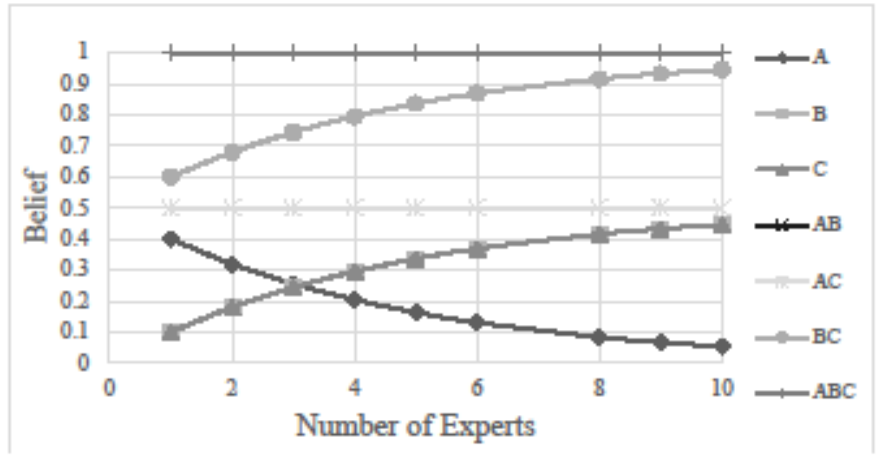

771 Fig. 4. Joint Belief Power Sets with 40\% Extra Confidence in Combined Events 772 